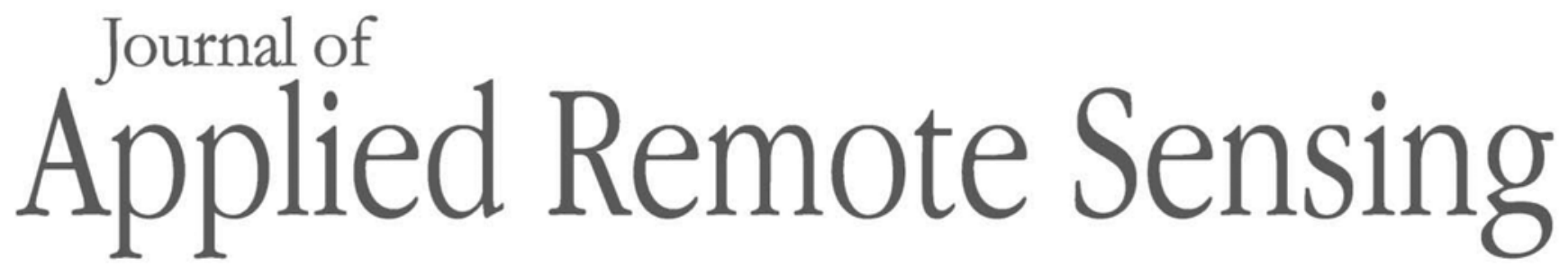

RemoteSensing.SPIEDigitalLibrary.org

\title{
Inverse synthetic aperture radar imaging of targets with nonsevere maneuverability based on the centroid frequency chirp rate distribution
}

Li Yanyan

$\mathrm{Su} \mathrm{TaO}$

Zheng Jibin

He Xuehui

\section{SPIE.}




\title{
Inverse synthetic aperture radar imaging of targets with nonsevere maneuverability based on the centroid frequency chirp rate distribution
}

\author{
Li Yanyan,* Su Tao, Zheng Jibin, and He Xuehui \\ Xidian University, School of Electronic Engineering, 2 South Taibai Road, Xi' an 710071, China
}

\begin{abstract}
For inverse synthetic aperture radar (ISAR) imaging of targets with nonsevere maneuverability, the Doppler frequencies of scatterers are actually time-varying and azimuth echoes of a range cell have to be modeled as multicomponent linear frequency modulation (LFM) signals after the range alignment and the phase adjustment. In ISAR imaging with the LFM signal model, the chirp rate deteriorates the target image and an effective parameter estimation algorithm is required. By employing a symmetric instantaneous self-correlation function and the modified scaled Fourier transform, an effective parameter estimation algorithm, known as the centroid frequency chirp rate distribution (CFCRD), is proposed and applied to ISAR imaging. Compared to two representative parameter estimation algorithms, the modified Wigner-Ville distribution and the Lv's distribution, the proposed CFCRD can acquire a higher antinoise performance without spectrum aliasing and brute-force searching. Through simulations and analyses of the synthetic radar data and the real radar data, we verify the effectiveness of CFCRD and the corresponding ISAR imaging algorithm. (C) The Authors. Published by SPIE under a Creative Commons Attribution 3.0 Unported License. Distribution or reproduction of this work in whole or in part requires full attribution of the original publication, including its DOI. [DOI: 10.1117/1.JRS.9.095065]
\end{abstract}

Keywords: inverse synthetic aperture radar; linear frequency modulation signal; modified scaled Fourier transform; centroid frequency chirp distribution.

Paper 15329 received May 5, 2015; accepted for publication Jul. 8, 2015; published online Aug. 12, 2015.

\section{Introduction}

Inverse synthetic aperture radar (ISAR) imaging has attracted the attention of radar researchers in the past three decades due to its all-weather suitability and day and night availability. ${ }^{1-4}$ The primary steps for ISAR imaging are the range alignment (compensating the translational and rotational range migrations) $)^{5-8}$ and the phase adjustment (removing the Doppler phase induced by the translation). ${ }^{9,10}$ Then the traditional range-Doppler (RD) method can be used to obtain the well-focused ISAR image for targets with smooth motions. However, for targets with nonsevere maneuverability, the traditional RD algorithm cannot work due to the time-varying Doppler frequencies of the scatterers. This ISAR imaging problem has motivated the research on the range-instantaneous Doppler (RID) technique ${ }^{11,12}$ and the range instantaneous chirp rate (RIC) technique. ${ }^{13,14}$

Based on the RID technique or RIC technique, azimuth echoes of targets with nonsevere maneuverability can be modeled as multicomponent linear frequency modulation (LFM) signals. In Refs. 11 and 12, the real radar data and the synthetic data have been utilized to validate the LFM signal model for ISAR imaging of targets with nonsevere maneuverability. For ISAR imaging with the LFM signal model, the chirp rate is identified as the cause of the image defocus and an effective parameter estimation algorithm is required. With regard to the parameter estimation of the LFM signal, many algorithms have been proposed. The cyclostationarity based algorithm, ${ }^{15}$ the Wigner-Ville distribution, ${ }^{16}$ the cubic phase function, ${ }^{17,18}$ and the ambiguity function ${ }^{19}$ are popular bilinear transforms, whose essences are to reduce the order of the LFM signal. Although the aforementioned bilinear algorithms have low computational cost and high

*Address all correspondence to: Li Yanyan, E-mail: liyanyan_xd@163.com 
resolution, the cross-term interference and the low antinoise performance limit their realistic applications. To resolve problems of the aforementioned bilinear algorithms, the integrated cubic phase function, ${ }^{20}$ the Radon-ambiguity transform, ${ }^{21}$ the keystone-Wigner transform, ${ }^{12,22}$ the fractional Fourier transform, ${ }^{23,24}$ and the modified discrete chirp Fourier transform ${ }^{25,26}$ are proposed. They employ the brute-force searching of the unknown chirp rate to accumulate the signal energy, which benefits the cross-term suppression and the antinoise performance. However, the brute-force searching will require a complicated radar system and a high computational cost $\left[O\left(M N \log _{2} N\right)\right]$, where $M$ is the searching time and is always much larger than the number of echoes $N$ in the high-resolution ISAR imaging application due to the high carrier frequency and the large frequency bandwidth. ${ }^{11,27,28}$

In order to overcome the problems of the aforementioned algorithms, ${ }^{15-26}$ the modified Wigner-Ville distribution and the Lv's distribution are proposed in Refs. 11 and 27, respectively. These two algorithms eliminate the brute-force searching and only require complex multiplication, the fast Fourier transform (FFT), and the inverse FFT to complete the signal energy accumulation. However, challenges still exist for these two algorithms and include (1) the modified Wigner-Ville distribution has a low antinoise performance and cannot avoid the problem of spectrum aliasing and (2) although the Lv's distribution can use the redundancy information to avoid spectrum aliasing, this is at the cost of the antinoise performance loss.

In this paper, an effective parameter estimation algorithm, known as the centroid frequency chirp rate distribution (CFCRD), is proposed by employing a novel symmetric instantaneous self-correlation function and the modified scaled Fourier transform (MSFT). The novel symmetric instantaneous self-correlation function benefits the antinoise performance and the MSFT helps the CFCRD avoid spectrum aliasing. The MSFT can be implemented with the FFT based chirp-z transform. Thus, the brute-force searching is eliminated in CFCRD. Through simulations and analyses, we demonstrate that, without spectrum aliasing, CFCRD can acquire a higher antinoise performance than the modified Wigner-Ville distribution and the Lv's distribution, which makes the CFCRD more practical. Thereafter, based on CFCRD, a novel ISAR imaging algorithm is presented and verified with synthetic data and real radar data.

The remainder of this paper is organized as follows. In Sec. 2, by employing the symmetric instantaneous self-correlation function and MSFT, CFCRD is proposed for the parameter estimation of the LFM signal. In addition, the cross-term and the selection criterion of the zoom factor are also analyzed for CFCRD. Section 3 gives analyses of the computational cost and the antinoise performance. Based on CFCRD, a novel ISAR imaging algorithm is presented for targets with nonsevere maneuverability in Sec. 4. Its application and the conclusion are given in Secs. 5 and 6, respectively.

\section{Centroid Frequency Chirp Rate Distribution for Parameters Estimation of Linear Frequency Modulation Signal}

The geometry for ISAR imaging used here is based on the model in Ref. 11. In this paper, we will not discuss the range alignment and the phase adjustment in detail, and will just focus on the processing of the Doppler frequency shift. With regard to the range alignment and the phase adjustment, we can refer the reader to Refs. 5-10 for corresponding processing algorithms. We assume that all scatterers of the target have been corrected into the right range cell after the range alignment and the phase adjustment, and the coupling between the envelope and the Doppler frequency ${ }^{29-31}$ will not be discussed in this paper. Assume the number of scatterers in the $a^{\prime}$ th range cell is $P$ and the azimuth echo takes the form of multicomponent LFM signals

$$
s(t)=\sum_{p=1}^{P} A_{p} \exp \left[j \phi_{p}(t)\right]+z(t)=\sum_{p=1}^{P} A_{p} \exp \left[j 2 \pi\left(f_{p} t+\frac{1}{2} \gamma_{p} t^{2}\right)\right]+z(t),
$$

where $t$ denotes the slow time, and $A_{p}$ and $\phi_{p}(t)$ are the amplitude and the signal phase of the $p$ 'th scatterer, respectively. $z(t)$ is additive complex white Gaussian noise with a variance of $\delta^{2}$. $f_{p}$ and $\gamma_{p}$ denote the centroid frequency and the chirp rate, respectively. According to Ref. $11, f_{p}$ and $\gamma_{p}$ correspond to the coordinate. Thus, different scatterers correspond to different $f_{p}$ and $\gamma_{p}$, and it is necessary to estimate the centroid frequency and the chirp rate for different scatterers. 
In ISAR imaging with the LFM signal model, the chirp rate causes the Doppler frequency shift and deteriorates the image quality. Thus, in order to reconstruct a well-focused ISAR image, the CFCRD is proposed in this section by employing a novel symmetric instantaneous self-correlation function and the MSFT. The following three sections will discuss the principle of CFCRD, the cross-term suppression, and the selection criterion of the zoom factor, respectively.

\subsection{Centroid Frequency Chirp Rate Distribution with Multicomponent Linear Frequency Modulation Signal}

Based on the format of Eq. (1) and the analyses in Ref. 11, a novel symmetric instantaneous selfcorrelation function is defined as

$$
R_{s}(t, \tau)=s(t+\tau) s^{*}(t-\tau)=\sum_{p=1}^{P} A_{p}^{2} \exp \left[j 2 \pi\left(2 f_{p} \tau+2 \gamma_{p} t \tau\right)\right]+R_{s, \text { cross }}(t, \tau)+Z_{R, \text { noise }}(t, \tau),
$$

where $*$ and $\tau$ denote the complex conjugation and the lag-time variable, respectively. $R_{s, \text { cross }}(t, \tau)$ and $Z_{R \text {,noise }}(t, \tau)$ denote the cross-term and the noise after the symmetric instantaneous self-correlation function, respectively.

It is worthwhile noting that the symmetric instantaneous self-correlation function defined in Eq. (2) is different from that in Ref. 11. As we know, due to the bilinearity, half of the energy of the original signal will be lost, ${ }^{32,33}$ which can be seen from Fig. 1(a). Thus, the symmetric instantaneous self-correlation function defined in Ref. 11 has a $3 \mathrm{~dB}$ antinoise performance loss. However, if there are enough samplings for the symmetric instantaneous self-correlation function as defined in Ref. 11, the antinoise performance loss may be saved. References 27 and 28 indicate that the previously processed data are useful and can be used as the redundancy information. Thus, for the symmetric instantaneous self-correlation function defined in Eq. (2), the previously processed data are used as the redundancy information to save the antinoise performance loss, which is shown in Fig. 1(b) (red triangles correspond to the data obtained with the previously processed data).

In the exponential phase term of Eq. (2), a linear coupling exists between $t$ and $\tau$. In order to remove the coupling, Ref. 11 proposes the scaled Fourier transform. However, because the estimation range of the chirp rate is fixed, spectrum aliasing easily occurs on the scaled Fourier

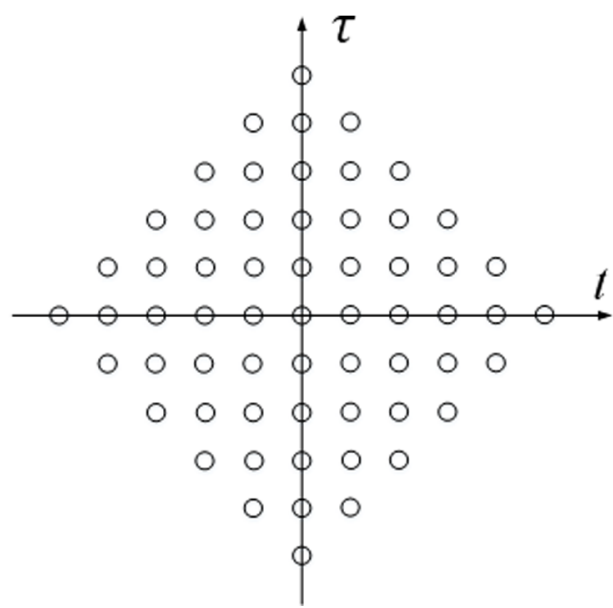

(a)

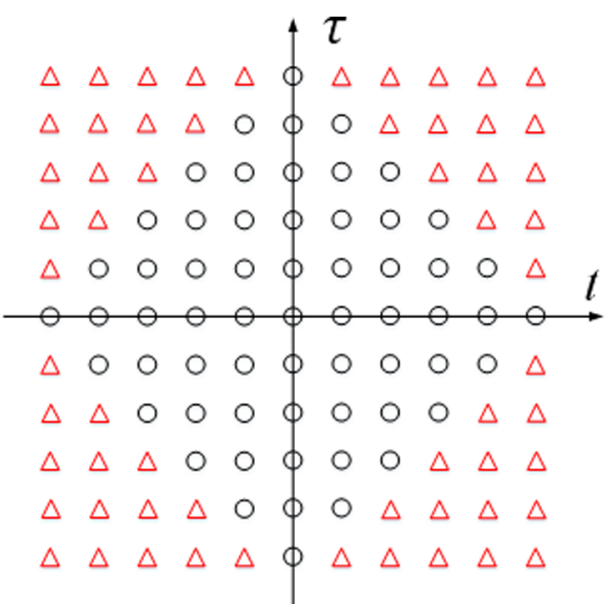

(b)

Fig. 1 Symmetric instantaneous self-correlation functions defined in Ref. 11 and Eq. (2): (a) symmetric instantaneous self-correlation function defined in Ref. 11 and (b) symmetric instantaneous self-correlation function defined in Eq. (2). 
transform and disables the modified Wigner-Ville distribution. Here, in order to remove the coupling without spectrum aliasing, we propose MSFT, which takes the form

$$
T_{S}\left(\tau, f_{t \tau}, \xi\right)=\operatorname{MSFT}_{t \tau}\left[R_{s}(t, \tau)\right]=\int_{t \tau} R_{s}(t, \tau) \exp \left[-j 2 \pi f_{t \tau} \xi t \tau\right] d t \tau,
$$

where $\operatorname{MSFT}_{t \tau}(\cdot)$ denotes the MSFT operator. Because of the introduction of the zoom factor $\xi$, the estimation range of the chirp rate can be enlarged and the problem of the spectrum aliasing will be resolved. In order to distinguish it from the scaled Fourier transform, we define it as MSFT. $f_{t \tau}$ is the frequency domain with respect to $t \tau$. $\xi$ denotes a zoom factor, which is used to solve the spectrum aliasing. The selection criterion of the zoom factor will be discussed in Sec. 2.3. Obviously, when $\xi=1$, the scaled Fourier transform proposed in Ref. 11 is only a special case of MSFT.

Substituting Eq. (2) into Eq. (3), we have the lag-time chirp rate distribution

$$
T_{s}\left(\tau, f_{t \tau}, \xi\right)=\sum_{p=1}^{P} A_{p}^{2} \exp \left(j 4 \pi f_{p} \tau\right) \delta\left(f_{t \tau}-\frac{2 \gamma_{p}}{\xi}\right)+T_{s, \text { cross }}\left(\tau, f_{t \tau}, \xi\right)+T_{R, \text { noise }}\left(\tau, f_{t \tau}, \xi\right),
$$

where $T_{s, \text { cross }}\left(\tau, f_{t \tau}, \xi\right)$ and $T_{R \text {,noise }}\left(\tau, f_{t \tau}, \xi\right)$ denote the cross-term and the noise after MSFT, respectively.

After MSFT, the coupling is eliminated and the signal energy peaks along the beeline $f_{t \tau}-2 \gamma_{p} / \xi=0$. Applying FFT to $T_{s}\left(\tau, f_{t \tau}, \xi\right)$ along the $\tau$ dimension, we get the CFCRD

$$
\begin{aligned}
& D_{s}\left(f_{\tau}, f_{t \tau}, \xi\right)=\operatorname{FFT}_{\tau}\left[T_{s}\left(\tau, f_{t \tau}, \xi\right)\right] \\
& \quad=\sum_{p=1}^{P} A_{p}^{2} \delta\left(f_{\tau}-2 f_{p}\right) \delta\left(f_{t \tau}-\frac{2 \gamma_{p}}{\xi}\right)+D_{s, \text { cross }}\left(f_{\tau}, f_{t \tau}, \xi\right)+D_{R, \text { noise }}\left(f_{\tau}, f_{t \tau}, \xi\right),
\end{aligned}
$$

where $\operatorname{FFT}_{\tau}(\cdot)$ denotes the FFT operator. $f_{\tau}$ is the frequency domain with respect to $\tau$. $D_{s, \text { cross }}\left(f_{\tau}, f_{t \tau}, \xi\right)$ and $D_{R, \text { noise }}\left(f_{\tau}, f_{t \tau}, \xi\right)$ denote the cross-term and the noise after the FFT operation, respectively.

In Eq. (5), each scatterer corresponds to a sole peak at the point $\left(2 f_{p}, 2 \gamma_{p} / \xi\right)$ on the centroid frequency and the chirp rate plane. Thus, if the cross-term does not accumulate as the self-term (this will be verified in the next section) and the CFCRD has a high antinoise performance (this will be verified in Sec. 3), parameters $f_{p}$ and $\gamma_{p}$ can be estimated as $\left(f_{\tau}^{\prime} / 2, \xi f_{t \tau}^{\prime} / 2\right)$ with the peak detection technique. ${ }^{11,27}$

The MSFT defined in Eq. (3) can be implemented with the FFT based chirp-z transform and we can refer to Appendix A for its implementation. Thus, just as with the modified Wigner-Ville distribution and the Lv's distribution, our proposed CFCRD can also complete the parameters' estimation of the LFM signal without the brute-force searching. Eliminations of the brute-force searching procedure benefit the radar system complexity. ${ }^{27}$ In the next two sections, the crossterm and the selection criterion of the zoom factor will be analyzed for CFCRD.

\subsection{Cross-Term Analysis}

Due to the bilinearity of the symmetric instantaneous autocorrelation function, the cross-term $D_{s, \text { cross }}\left(f_{\tau}, f_{t \tau}, \xi\right)$ appears in the CFCRD and may influence the detection of the self-term. Thus, in Appendix B, we analyze characteristics of the cross-term and the obtained lemma are listed below.

Lemma. Let $\nabla f_{p_{1}, p_{2}}=f_{p_{1}}+f_{p_{2}}, \Delta f_{p_{1}, p_{2}}=f_{p_{1}}-f_{p_{2}}, \nabla \gamma_{p_{1}, p_{2}}=\left(\gamma_{p_{1}}+\gamma_{p_{2}}\right) / 2, \Delta \gamma_{p_{1}, p_{2}}=$ $\left(\gamma_{p_{1}}-\gamma_{p_{2}}\right) / 2, \tilde{f}_{t \tau}=f_{t \tau}-2 \nabla \gamma_{p_{1}, p_{2}} / \xi$, and $\tilde{f}_{\tau}=f_{\tau}-\nabla f_{p_{1}, p_{2}}$. The corresponding cross-term can be expressed as 
1. for $\gamma_{p_{1}}=\gamma_{p_{2}}, f_{p_{1}} \neq f_{p_{2}}$, and $\tilde{f}_{t \tau}=0$

$$
D_{s, \operatorname{cros}}\left(f_{\tau}, f_{t \tau}, \xi\right)=0,
$$

2. for $\gamma_{p_{1}}=\gamma_{p_{2}}, f_{p_{1}} \neq f_{p_{2}}$, and $\tilde{f}_{t \tau} \neq 0$

$$
D_{s, \operatorname{cros}}\left(f_{\tau}, f_{t \tau}, \xi\right)=2 A_{p_{1}} A_{p_{2}} \frac{\Delta f_{p_{1}, p_{2}}}{\xi \tilde{f}_{t \tau}^{2}} \cos \left(2 \pi \frac{\Delta f_{p_{1}, p_{2}}}{\xi \tilde{f}_{t \tau}} \tilde{f}_{\tau}\right),
$$

3. for $\gamma_{p_{1}} \neq \gamma_{p_{2}}$

$$
\begin{aligned}
D_{s, \text { cros }}\left(f_{t \tau}, f_{\tau}, \xi\right)= & \frac{8 A_{p_{1}} A_{p_{2}} \xi\left|\tilde{f}_{\tau} \Delta \gamma_{p_{1}, p_{2}}\right|}{\left.\left|\xi^{2} \tilde{f}_{t \tau}^{2}+\Delta \gamma_{p_{1}, p_{2}}^{2}\right|\right|^{3 / 2}} \cos \left\{\operatorname{sgn}\left(\Delta \gamma_{p_{1}, p_{2}}\right) \frac{\pi}{4}[1\right. \\
& \left.\left.+\operatorname{sgn}\left(\xi^{2} \tilde{f}_{t}^{2}+\Delta \gamma_{p_{1}, p_{2}}^{2}\right)\right]+\frac{2 \tilde{f}_{\tau}^{2} \Delta \gamma_{p_{1}, p_{2}}}{\xi^{2} \tilde{f}_{t \tau}^{2}+\Delta \gamma_{p_{1}, p_{2}}^{2}}-\frac{\Delta f_{p_{1}, p_{2}}^{2}}{2 \Delta \gamma_{p_{1}, p_{2}}}\right\} .
\end{aligned}
$$

Obviously, under multicomponent LFM signals, the cross-term of the CFCRD cannot accumulate as the self-term and has a cosine structure. Thus, for the self-term, the effect of the crossterm can be neglected. In the following, an example will be given to show how the CFCRD accumulates the self-term and suppresses the cross-term.

Example 1: We consider two LFM signals denoted by $A u 1$ and $A u 2$. The pulse repetition frequency (PRF) and the number of effective integration pulses $N$ are $128 \mathrm{~Hz}$ and 128 , respectively. Parameters of $A u 1$ and $A u 2$ are set as follows: $A_{1}=1, f_{1}=10 \mathrm{~Hz}, \gamma_{1}=20 \mathrm{~Hz} / \mathrm{s}$; $A_{2}=1, f_{2}=-10 \mathrm{~Hz}, \gamma_{2}=100 \mathrm{~Hz} / \mathrm{s}$. Figure 2 gives the simulation results.

With the FFT operation along the slow time axis of $R_{s}(t, \tau)$, Fig. 2(a) gives the lag-timeDoppler frequency distribution. Obviously, the coupling between $t$ and $\tau$ induces inclined

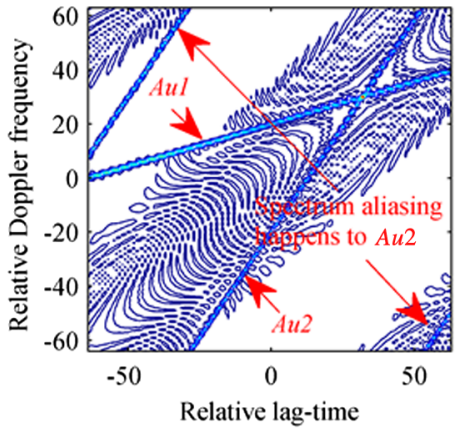

(a)

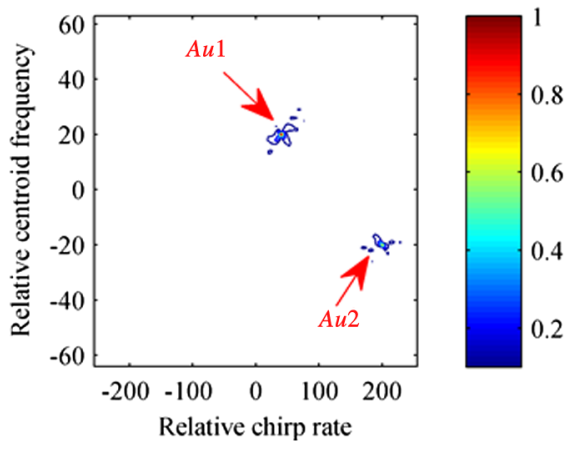

(c)
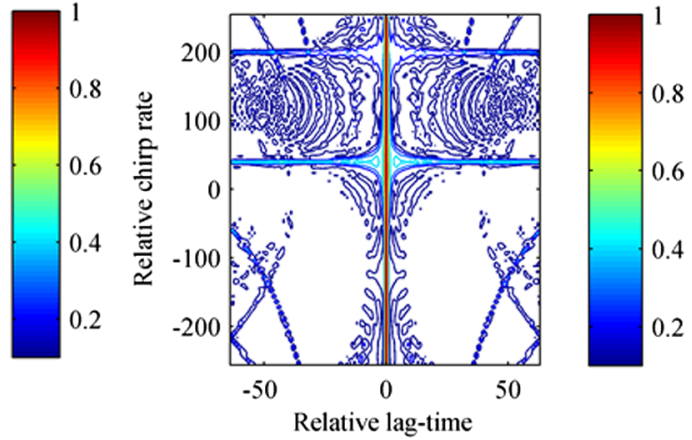

(b)

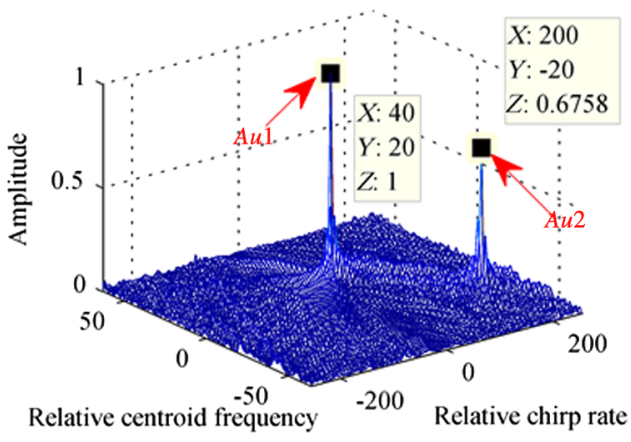

(d)

Fig. 2 Simulation results of example 1: (a) contour of the lag-time Doppler frequency distribution, (b) contour of the lag-time chirp rate distribution after the modified scaled Fourier transform, (c) contour of the centroid frequency chirp rate distribution (CFCRD), and (d) stereogram of CFCRD. 
lines. It is worthwhile noting that, as shown in Fig. 2(a), the spectrum aliasing happens to $A u 2$ due to its great chirp rate. We apply the MSFT of Eq. (3) to remove the coupling of $R_{s}(t, \tau)$ with the zoom factor $\xi=4$ (this zoom factor can solve the problem of spectrum aliasing and its selection criterion will be discussed in Sec. 2.3). In Fig. 2(b), after MSFT, the spectrum aliasing is eliminated and the self-term is corrected into beelines. However, the MSFT does not work on the cross-term, which is still typically dispersed on the lag-time chirp rate plane. Performing the FFT operation along the lag-time axis of Fig. 2(b), we obtain Fig. 2(c), where only the self-term accumulates into sole peaks. Figure 2(d) gives the stereogram of Fig. 2(c). In Fig. 2(d), with the peak detection technique, $\left(\gamma_{1}, f_{1}\right)$ and $\left(\gamma_{2}, f_{2}\right)$ can be estimated as $(20 \mathrm{~Hz} / \mathrm{s}, 10 \mathrm{~Hz})$ and $(100 \mathrm{~Hz} / \mathrm{s},-10 \mathrm{~Hz})$, respectively.

The aforementioned analyses and simulations are under the situation of multicomponent LFM signals with the same amplitude. However, in realistic applications, amplitudes are usually different. ${ }^{27}$ In general, weak LFM signals may be submerged in the residual cross-term generated by strong LFM signals. Therefore, under multicomponent LFM signals with different amplitudes, similar to other parameter estimation algorithms, the clean technique ${ }^{23-25}$ should be performed to separate weak LFM signals from strong LFM signals.

\subsection{Selection Criterion of the Zoom Factor}

For the MSFT proposed in Eq. (3), the zoom factor $\xi$ is introduced to avoid the spectrum aliasing and eliminate the estimation error. In this section, we will employ the analytical method utilized in Ref. 34 to discuss the selection criterion of the zoom factor $\xi$ in detail.

After the energy accumulation, the unknown chirp rate $\gamma_{p}$ can be estimated as $\hat{\gamma}_{p}$ through the peak detection technique. ${ }^{34}$

$$
\hat{\gamma}_{p}=f_{t \tau} \xi / 2
$$

Considering the sampling frequency is $F_{\Upsilon}+1$ ( $F_{\Upsilon}$ is even integer), we can obtain the range of $f_{t \tau}$ for the CFCRD

$$
-F_{\Upsilon} / 2 \leq f_{t \tau} \leq F_{\Upsilon} / 2 .
$$

In order to avoid spectrum aliasing, it is assumed that $\left|\gamma_{p}\right| \leq F_{Y}^{2} / N .^{18}$ Thus, based on Eq. (10), the ranges of the zoom factor should be

$$
\xi \geq 4 F_{\Upsilon} / N \text {. }
$$

This means that if the zoom factor satisfies Eq. (11), the spectrum aliasing and the estimation error can be totally eliminated. However, according to Eq. (9), a smaller zoom factor can be utilized to increase the estimation accuracy. Therefore, the selection criterion of the zoom factor should combine Eq. (11) with the requirement of the estimation precision in realistic ISAR imaging. Based on the analyses in Ref. 34, in order to obtain a balance between Eq. (11) and the estimation precision, ${ }^{11,29}$ we suggest $\xi=4 F_{\Upsilon} / N$ for CFCRD.

\section{Antinoise Performance Analysis and Computational Cost}

In this section, we will analyze the antinoise performance and the computational cost of the CFCRD, which play important roles in the parameters' estimation. ${ }^{27,28,35-37}$ The modified Wigner-Ville distribution algorithm and the Lv's distribution algorithm are chosen as references.

\subsection{Antinoise Performance}

In this section, the input-output signal-to-noise ratio (SNR) $)^{35-37}$ and the mean square error ${ }^{26,37-39}$ are utilized to evaluate the antinoise performance of CFCRD.

Example 2: A monocomponent LFM signal $B u$, which is contaminated with the zero-mean complex white Gaussian noise, is taken into account in this example. The PRF and the number of 


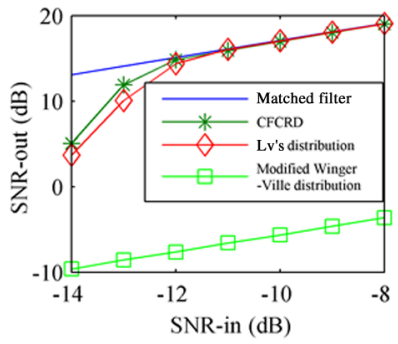

(a)

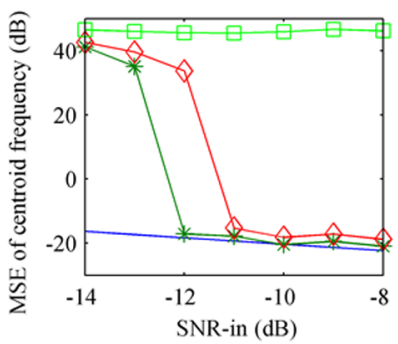

(b)

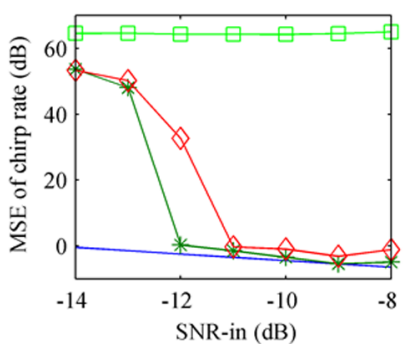

(c)

Fig. 3 Analysis of the antinoise performance: (a) input-output signal-to-noise ratio performance, (b) mean square error (MSE) of centroid frequency estimation, and (c) MSE of chirp rate estimation.

effective integration pulses $N$ are $256 \mathrm{~Hz}$ and 256, respectively. The signal parameters are $A=1$, $f=60 \mathrm{~Hz}, \gamma=200 \mathrm{~Hz} / \mathrm{s}$. The tested input SNRs are $\mathrm{SNR}_{\text {in }}=[-14:-8]$, and 100 trials are performed for each $\mathrm{SNR}_{\text {in }}$ value. According to the selection criterion of the zoom factor, we set the zoom factor to four in this example. Figure 3 gives the simulation results.

The input-output SNR comparison is shown in Fig. 3(a), where the result of the matched filter for the LFM signal $B u$ in example 2 is also shown with the solid line. The chirp rate of $B u$ exceeds the range of the traditional scaled Fourier transform for the modified Wigner-Ville distribution. Thus, the spectrum aliasing appears and the estimation error happens to the modified Wigner-Ville distribution algorithm in Fig. 3(a). For the Lv's distribution, the problem of the spectrum aliasing can be avoided by increasing the value of the parameter $h$ according to Eq. (7) in Ref. 27. According to the principle of the Lv's distribution, the parameter $h$ should be set to 2 to avoid the spectrum aliasing. However, due to the limitation of $a h=1$, the time delay $a=1 / 2$ influences the antinoise performance. ${ }^{28}$ In Figs. 3(b)-3(c), we can see that the threshold SNR of the Lv's distribution is $-11 \mathrm{~dB}$. In CFCRD, the novel symmetric instantaneous self-correlation function benefits the antinoise performance, and the antinoise performance will not be influenced by the variety of the value of $\xi$. Therefore, the threshold SNR of CFCRD is $-12 \mathrm{~dB}$, which can make CFCRD more suitable for realistic applications than the modified WignerVille distribution and the Lv's distribution.

The observed mean square errors (MSEs) for the centroid frequency and the chirp rate are plotted in Figs. 3(b)-3(c) as a function of SNR, respectively. The corresponding Cramer-Rao bounds (CRBs) are also shown in solid lines in Figs. 3(b)-3(c), and the expressions of the corresponding CRBs can be obtained from Refs. 40 and 41. Figures 3(b) and 3(c) show that, as expected, both MSEs of the centroid frequency and chirp rate estimations are inversely proportional to the input SNRs. The MSEs of the centroid frequency and chirp rate estimation are close to $\mathrm{CRB}$ when $\mathrm{SNR} \geq-12 \mathrm{~dB}$, which further validates the results shown in Fig. 3(a).

\subsection{Computational Cost}

In Sec. 3.1, we analyze the antinoise performance of CFCRD and demonstrate that CFCRD can obtain a higher antinoise performance than the modified Wigner-Ville distribution and the Lv's distribution. In this section, the computational cost of CFCRD will be analyzed.

For the modified Wigner-Ville distribution, ${ }^{11}$ its main implementation procedures include the defined self-correlation function $\left[O\left(N^{2}\right)\right]$, the scaled Fourier transform $\left[O\left(N^{2} \log _{2} N\right)\right]$, and the FFT operation along the lag-time axis $\left[O\left(N^{2} \log _{2} N\right)\right]$. Thus, the computational cost of the modified Winger-Ville distribution is the order of $O\left(N^{2} \log _{2} N\right)$. For the Lv's distribution, ${ }^{27}$ its main implementation procedures include the defined self-correlation function $\left[O\left(N^{2}\right)\right]$, the keystone transform $\left[O\left(N^{2} \log _{2} N\right)\right]$, and the FFT operation along the lag-time axis $\left[O\left(N^{2} \log _{2} N\right)\right]$. Thus, the computational cost of the Lv's distribution is the order of $O\left(N^{2} \log _{2} N\right)$. The main implementation procedures of CFCRD include the defined parameter symmetric self-correlation function $\left[O\left(N^{2}\right)\right]$, the MSFT $\left[O\left(N^{2} \log _{2} N\right)\right]$, and the FFT operation along the lag-time axis $\left[O\left(N^{2} \log _{2} N\right)\right]$. Therefore, the computational cost of CFCRD is in the order of $O\left(N^{2} \log _{2} N\right)$. 
Table 1 Computational cost.

\begin{tabular}{lccc}
\hline \hline Estimation algorithms & Modified Winger-Ville distribution & Lv's distribution & $\begin{array}{c}\text { Centroid frequency chirp } \\
\text { rate distribution }\end{array}$ \\
\hline Computational cost & $O\left(N^{2} \log _{2} N\right)$ & $O\left(N^{2} \log _{2} N\right)$ & $O\left(N^{2} \log _{2} N\right)$ \\
\hline \hline
\end{tabular}

Table 1 gives the computational costs of these three algorithms. According to Table 1, we know that the computational cost of CFCRD is in the same order as those of the modified Winger-Ville distribution and the Lv's distribution.

\section{Inverse Synthetic Aperture Radar Imaging Algorithm Based on Centroid Frequency Chirp Rate Distribution}

With the simulations and analyses in Secs. 2 and 3, we know that, considering the elimination of the brute-force searching, the cross-term suppression, the settlement of the spectrum aliasing, the computational cost, and the antinoise performance, our proposed CFCRD is more practical than the modified Winger-Ville distribution and the Lv's distribution. Therefore, in this section, by employing CFCRD, a novel imaging algorithm is proposed for targets with nonsevere maneuverability and its implementation is illustrated as follows.

Step 1: Complete the range compression of radar echoes with the matched filter

$$
H(\hat{t})=\operatorname{rect}\left(\hat{t} / T_{s}\right) \exp \left(j \pi \gamma \hat{t}^{2}\right),
$$

where

$$
\operatorname{rect}[x]= \begin{cases}1, & |x| \leq 1 / 2 \\ 0, & |x|>1 / 2\end{cases}
$$

$\hat{t}, T_{s}$, and $\gamma$ are the fast time, the pulse width, and the frequency modulation rate, respectively.

Step 2: Compensate the translational range migration, the rotational range migration, and the Doppler spread induced by the translation.

Step 3: Get the data $s_{l}(t)$ of the $l^{\prime}$ th (where $1 \leq l \leq L$ and $L$ is the number of range cells) range cell.

Step 4: Apply CFCRD to the data $s_{l}(t)$, and then the peak detection technique ${ }^{29,32}$ can be utilized to estimate the magnitude $A_{p}$, the centroid frequency $f_{p}$, and the chirp rate $\gamma_{p}$

$$
\left(A_{p}^{\prime}=\frac{D^{\prime}}{N^{2}}, f_{p}^{\prime}=\frac{f_{\tau}}{2}, \gamma_{p}^{\prime}=\frac{f_{t \tau} \xi}{2}\right)=\underset{\left(f_{\tau}, f_{t \tau}\right)}{\operatorname{argmax}}\left|D_{s_{l}}\left(f_{\tau}, f_{t \tau}, \xi\right)\right|,
$$

where $D^{\prime}$ denotes the amplitude of the peak. $f_{p}^{\prime}$ and $\gamma_{p}^{\prime}$ are estimations of the centroid frequency and the chirp rate for the $p$ 'th LMF of $s_{l}(t)$. argmax denotes the argument that maximizes. $D_{s_{l}}\left(f_{\tau}, f_{t \tau}, \xi\right)$ denotes the result after applying CFCRD on $s_{l}(t)$.

Step 5: Subtract the estimated $p$ 'th LFM from the original signal $s_{l}(t)$

$$
s_{l}(t)=s_{l}(t)-A_{p}^{\prime} \exp \left[j 2 \pi\left(f_{p}^{\prime} t+\frac{1}{2} \gamma_{p}^{\prime} t^{2}\right)\right] .
$$

Step 6: Repeat steps 4 and 5 until the residual signal energy $E$ is less than $E_{H}$ (say, $5 \%$ of the original signal) ${ }^{29,32}$ which is an energy threshold.

Step 7: If $l<L$, set $l=l+1$ and repeat steps 3 to 6 until $l=L$.

Step 8: Reconstruct the azimuth echo as $s_{l}(t)=\sum_{p=1}^{P} A_{p}^{\prime} \exp \left(j 2 \pi f_{p}^{\prime} t\right), 1 \leq l \leq L$, and finally, obtain the ISAR imaging via FFT operation.

Above is the proposed ISAR imaging algorithm based on CFCRD. With the estimated parameters, we can reconstruct the well-focused ISAR image for targets with nonsevere maneuverability. 


\section{Verification of the Proposed Inverse Synthetic Aperture Radar Imaging Algorithm}

\subsection{Inverse Synthetic Aperture Radar Imaging with Synthetic Ship Model}

In this section, ISAR imaging algorithms, which utilize the modified Winger-Ville distribution, the Lv's distribution, and CFCRD are compared. Similar to Refs. 12, 14, 34, and 37-39, a fluctuating ship shown in Fig. 4(a) is modeled as a set of ideal scatterers. The unit vector of the radar line of sight is $(2 / \sqrt{69},-8 / \sqrt{69}, 1 / \sqrt{69})$. Figure 4 (b) shows the result of the ideal RD algorithm under the absence of the range migration and the Doppler frequency, while Fig. 4(c) is the result of the RD algorithm with the Doppler frequency shift after the motion compensation. The parameters of the radar and the ship model are listed in Table 2.

The comparison between Figs. 4(b) and 4(c) demonstrates that the existence of the chirp rate induces the Doppler frequency shift and degrades the quality of the ISAR image. Therefore, for ISAR imaging of targets with nonsevere maneuverability, we should estimate the chirp rate and compensate the corresponding Doppler frequency shift.

In Fig. 5, the ISAR imaging algorithms based on the modified Wigner-Ville distribution and the Lv's distribution are utilized to compare with the ISAR imaging algorithm based on CFCRD under the situation of $\mathrm{SNR}_{\mathrm{in}}=-11 \mathrm{~dB}$. The zoom factor $\xi$ of CFCRD is set to 4 , and the parameter $h$ of the Lv's distribution is set to 2. The results of Fig. 5 are normalized, and the entropy of Eq. (15) is utilized as a criterion to measure the quality of the image $I(h, n)$ in Table $3 .^{34,37}$

$$
E N T=-\sum_{h=1}^{H} \sum_{n=1}^{N}|I(h, n)|^{2} \ln |I(h, n)|^{2} .
$$

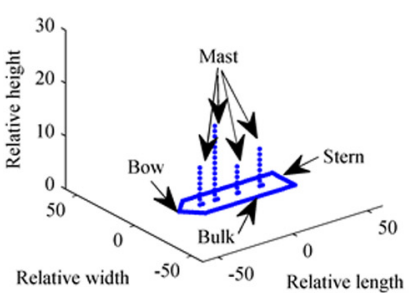

(a)

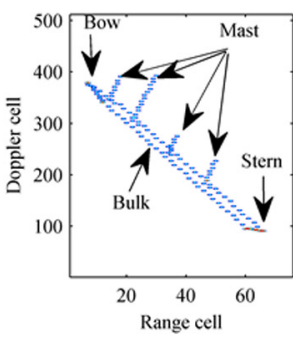

(b)
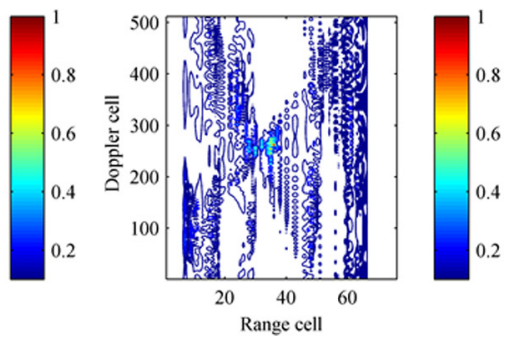

(c)

Fig. 4 Ship model and results of range-Doppler (RD) algorithm: (a) ship model, (b) ideal RD image, and (c) RD image with Doppler frequency shift.

Table 2 Radar parameters and moving parameters of the ship model.

\begin{tabular}{lccc}
\hline \hline Carrier frequency $f_{c}$ & $5 \mathrm{GHz}$ & Wave length $\lambda$ & $0.06 \mathrm{~m}$ \\
Bandwidth $B$ & $100 \mathrm{MHz}$ & Fast time sampling frequency $f_{s}$ & $100 \mathrm{MHz}$ \\
Pulse repetition frequency $F_{s}$ & $256 \mathrm{~Hz}$ & Effective echo pulses $N$ & 256 \\
\hline Translation coefficient & Velocity & Acceleration & \\
& $30 \mathrm{~m} / \mathrm{s}$ & $2 \mathrm{~m} / \mathrm{s}^{2}$ & $Z \mathrm{axis}$ \\
Rotating coefficient & $X \mathrm{axis}$ & $Y$ axis & $0.02 \mathrm{rad} / \mathrm{s}$ \\
Constant term & $0.03 \mathrm{rad} / \mathrm{s}$ & $0.01 \mathrm{rad} / \mathrm{s}$ & $0.06 \mathrm{rad} / \mathrm{s}^{2}$ \\
Acceleration term & $0.06 \mathrm{rad} / \mathrm{s}^{2}$ & $0.03 \mathrm{rad} / \mathrm{s}^{2}$ &
\end{tabular}




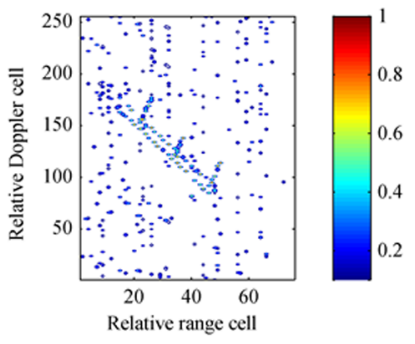

(a)

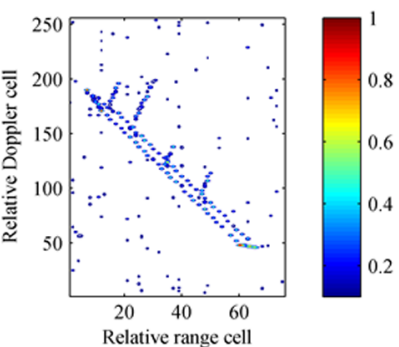

(b)

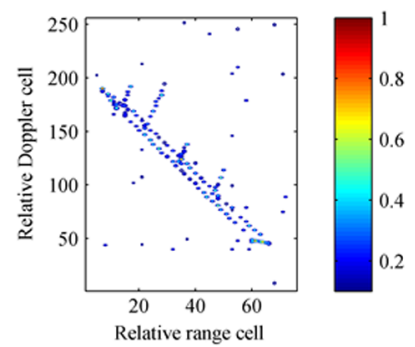

(c)

Fig. 5 Results of three inverse synthetic aperture radar (ISAR) imaging algorithms: (a) result of the modified Wigner-Ville distribution-based ISAR imaging algorithm, (b) result of the Lv's distributionbased ISAR imaging algorithm, and (c) result of the CFCRD-based ISAR imaging algorithm.

Table 3 Entropies of inverse synthetic aperture radar (ISAR) imaging in Fig. 5.

\begin{tabular}{lccc}
\hline \hline & Figure 5(a) & Figure 5(b) & Figure 5(c) \\
\hline Entropies & 109.2346 & 53.5553 & 40.4453 \\
\hline \hline
\end{tabular}

Figure 5(a) gives the result of the modified Wigner-Ville distribution-based ISAR imaging algorithm. Because the chirp rates of some scatterers exceed the estimation range of the traditional scaled Fourier transform, spectrum aliasing occurs and the Doppler frequency cannot be correctly estimated by the modified Winger-Ville distribution. Therefore, due to spectrum aliasing and a low $\mathrm{SNR}_{\mathrm{in}}$, the modified Wigner-Ville distribution-based ISAR imaging algorithm cannot reconstruct a well-focused image. The Lv's distribution-based ISAR imaging algorithm and the CFCRD-based ISAR imaging algorithm can both solve the spectrum aliasing and have a good antinoise performance. Thus, the entropies of Figs. 5(b) and 5(c) are lower than that of Fig. 5(a). Compared to Lv's distribution, CFCRD has a higher antinoise performance. Therefore, the quality of Fig. 5(c) is better than that of Fig. 5(b). The results of Fig. 5 demonstrate that the CFCRD-based ISAR imaging algorithm outperforms the modified Wigner-Ville distributionbased ISAR imaging algorithm and the Lv's distribution-based ISAR imaging algorithm.

\subsection{Inverse Synthetic Aperture Radar Imaging with Real Data}

In this section, the real data of a ship are utilized to validate the CFCRD-based ISAR imaging algorithm. For the Ku-band radar, the bandwidth is $240 \mathrm{MHz}$ and the PRF is $125 \mathrm{~Hz}$. The zoom factor of the CFCRD is set to 4. In simulations below, effective echoes are 125. Figure 6(a) presents the result after motion compensation. With the Wigner-Ville distribution, the time-frequency analysis of the 81st range cell is given in Fig. 6(b). Based on the result shown in Fig. 6(b) and the characteristic of the LFM signal, we know that the echoes of this ship should be modeled as multicomponent LFM signals.

The synthetic data have already been utilized to verify the effectiveness of CFCRD in the aforementioned sections. Here, before ISAR imaging with the real radar data, we utilize the real data of the 81st range cell to verify the effectiveness of CFCRD and the simulation results are shown in Fig. 7.

Figure 7(a) gives the lag-time Doppler frequency distribution after the FFT operation along the slow time axis of $R_{s}(t, \tau)$. Similar to example 1, an inclined line appears in Fig. 7(a). In order to correct the inclined line, MSFT is performed and the lag-time chirp rate distribution is shown in Fig. 7(b), where a beeline appears. On performing FFT operation along the lag-time axis of Fig. 7(b), we obtain the CFCRD in Fig. 7(c). Obviously, the signal energy accumulates into a sole peak. With the peak detection technique, the centroid frequency and the chirp rate are estimated as $-10.25 \mathrm{~Hz}$ and $-13 \mathrm{~Hz} / \mathrm{s}$, respectively. By compensating the Doppler frequency shift pertaining to the estimated chirp rate and performing an FFT, we obtain Fig. 7(d), where the result of the conventional RD algorithm is also shown. Due to the Doppler spread induced by the 


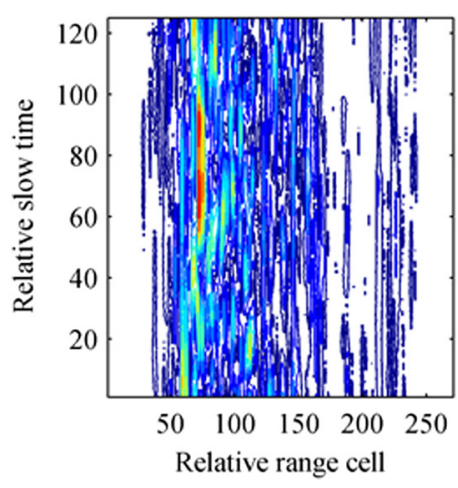

(a)

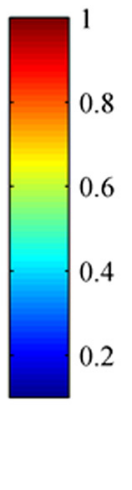

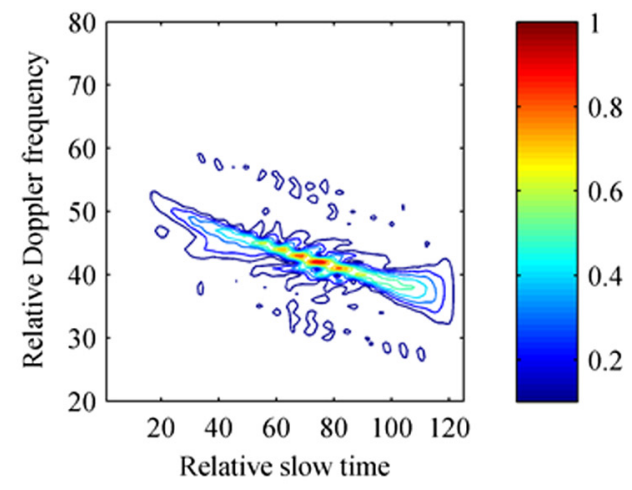

(b)

Fig. 6 Processing results of the real data: (a) azimuth echoes after the range alignment and the phase adjustment and (b) Wigner-Ville distribution of the 81th range cell.

chirp rate, the conventional RD algorithm cannot focus the signal energy to the correct Doppler frequency cell. It is known that several scatterers may exist in one range cell. Thus, steps 4 to 6 of the CFCRD-based ISAR imaging algorithm can be employed to relocate other potential scatterers in the 81 st range cell. The results of Fig. 7 demonstrate that the CFCRD works well for the real radar data of the fluctuating ship.

It is known that, due to the complicated and unknown realistic environment, we cannot control the characteristics of the received real data, such as the antinoise performance and the number of scatterers in each range cell. Thus, similar to Refs. 38 and 42, we utilize only the real radar data to verify whether the proposed ISAR imaging algorithm can work in realistic applications. The advantages of the proposed algorithm can be validated with synthetic data, such as simulations in Sec. 5.1. Figure 8 gives the ISAR imaging results, which are normalized.

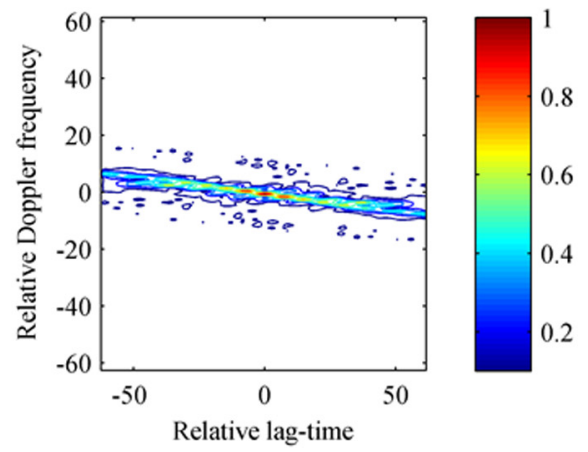

(a)

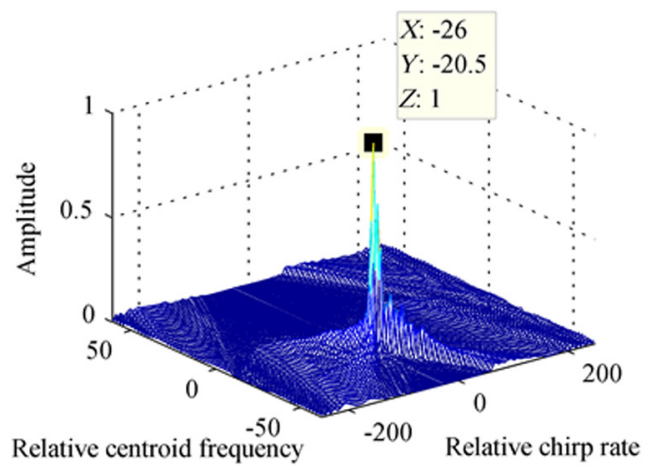

(c)

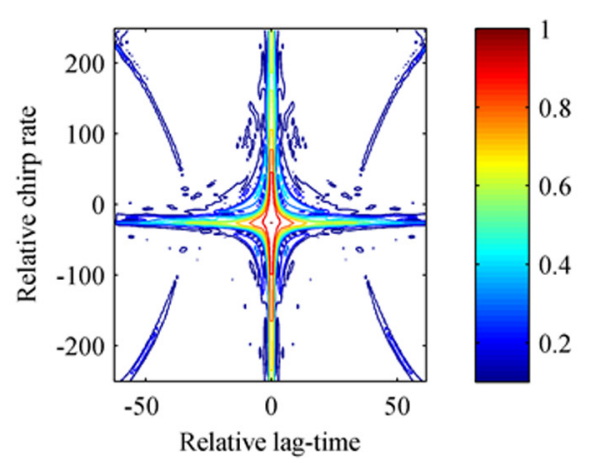

(b)

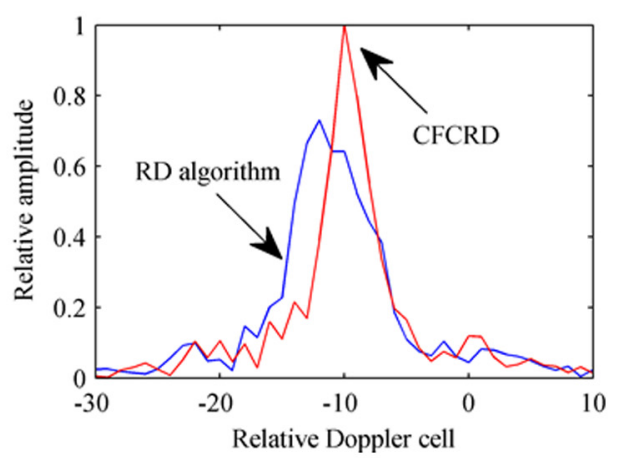

(d)

Fig. 7 Simulation results based on CFCRD: (a) contour of the lag-time Doppler frequency distribution, (b) contour of the lag-time chirp rate distribution, (c) stereogram of CFCRD, (d) conventional $\mathrm{RD}$ algorithm and the result after the Doppler spread compensation with CFCRD. 


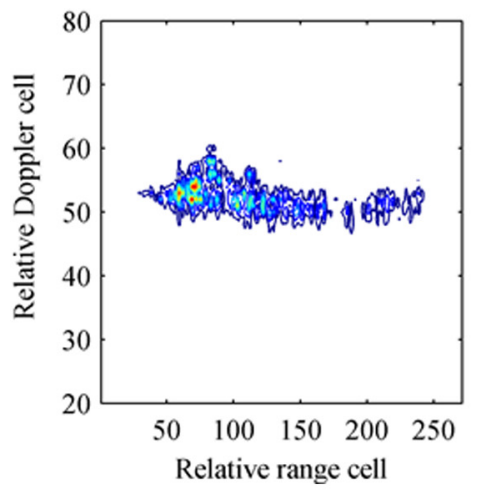

(a)

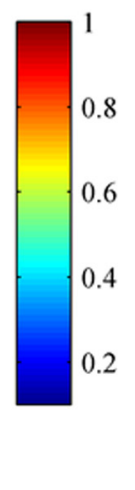

0.6

0.2

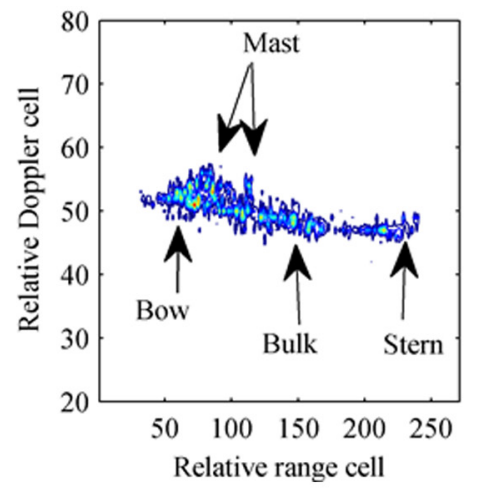

(b)

Fig. 8 ISAR imaging results with the real radar data: (a) result of the conventional RD algorithm and (b) result of the CFCRD-based ISAR imaging algorithm.

Table 4 Entropies of ISAR imaging in Fig. 8.

\begin{tabular}{lcc}
\hline \hline & Figure 8(a) & Figure 8(b) \\
\hline Entropies & 146.9266 & 98.9293 \\
\hline \hline
\end{tabular}

Figure 8(a) is the result of the conventional RD algorithm. Obviously, due to the Doppler spread induced by the chirp rate, the conventional RD algorithm cannot reconstruct a wellfocused image for the fluctuating ship. CFCRD can estimate parameters of multicomponent LFM signals with good cross-term suppression and a high antinoise performance. Thus, most scatterers of the fluctuating ship can be relocated correctly and few spurious scatterers appear in Fig. 8(b), where the mast, bow, stern, and bulk can be easily discerned. The entropies listed in Table 4 also demonstrate that the CFCRD-based ISAR imaging algorithm is suitable for realistic applications.

\section{Conclusion}

A nonsearching parameter estimation algorithm, referred to as CFCRD, is proposed by employing a symmetric instantaneous self-correlation function and MSFT. In this paper, the cross-term, the antinoise performance, the selection of the zoom factor, the implementation, and the computational cost of CFCRD are analyzed. Due to the symmetric instantaneous self-correlation function and MSFT, CFCRD can obtain a higher antinoise performance without a spectrum than the modified Winger-Ville distribution and the Lv's distribution. With the proposed CFCRD, an ISAR imaging algorithm is proposed. Thereafter, we utilize the synthetic data and the real data to validate the effectiveness of the CFCRD-based ISAR imaging algorithm.

\section{Appendix A}

We utilize the chirp-z transform to implement the modified scaled Fourier transform (MSFT). The details are as follows.

Consider the discrete format of Eq. (4).

$$
T_{d}\left(f_{t \tau}, n_{\tau}, \xi\right)=\sum_{n_{t}} A^{2} \exp \left\{j 2 \pi\left[2 f_{n} n_{\tau} T_{s}+\left(2 \gamma_{n}-f_{t \tau} \xi\right) n_{t} n_{\tau} T_{s}^{2}\right]\right\}
$$

where $n_{\tau}$ and $n_{t}$ correspond to $\tau$ and $t$, respectively; $T_{s}$ denotes the sampling interval. 
In order to reduce the computational cost, Eq. (16) can be rewritten as

$$
\begin{aligned}
T_{d}\left(f_{t \tau}, n_{\tau}, \xi\right)= & A^{2} \exp \left[j 2 \pi\left(2 f_{n} n_{\tau} T_{s}\right)\right] \exp \left(j 2 \pi \frac{-f_{t \tau}^{2}}{2} \xi n_{\tau} T_{s}^{2}\right) \\
& \cdot \sum_{n_{t}} A^{2} \exp \left\{j 2 \pi\left[\left(2 \gamma_{n} n_{t}-\frac{n_{t}^{2}}{2} \xi\right) n_{\tau} T_{s}^{2}\right]\right\} \exp \left[j 2 \pi \frac{\left(f_{t \tau}-n_{t}\right)^{2}}{2} \xi n_{\tau} T_{s}^{2}\right] .
\end{aligned}
$$

In Eq. (17), the item $T_{c}\left(f_{t \tau}, n_{\tau}, \xi\right)$ can be written as

$$
T_{c}\left(f_{t \tau}, n_{\tau}, \xi\right)=\sum_{n_{t}} A^{2} \exp \left\{j 2 \pi\left[\left(2 \gamma_{n} n_{t}-\frac{n_{t}^{2}}{2} \xi\right) n_{\tau} T_{s}^{2}\right]\right\} \exp \left[j 2 \pi \frac{\left(f_{t \tau}-n_{t}\right)^{2}}{2} \xi n_{\tau} T_{s}^{2}\right]
$$

It is obvious that $T_{c}\left(f_{t \tau}, n_{\tau}, \xi\right)$ can be regarded as a convolution. Thus, we can use fast Fourier transform (FFT) to implement it.

\section{Appendix B}

After FFT and MSFT operators, the cross-term $D_{s, \text { cros }}\left(f_{\tau}, f_{t \tau}, \xi\right)$, which influences the performance of CFCRD, still exists in $D_{s}\left(f_{\tau}, f_{\tau \tau}, \xi\right)$. Therefore, characteristics of the cross-term should be analyzed.

For a given $p_{1} \in[1, K-1]$ and $p_{2} \in\left[p_{1}+1, K\right]$, the cross-term in $R_{s, \text { cros }}(t, \tau)$ can be represented as

$$
\begin{aligned}
R_{p_{1}, p_{2}}(t, \tau)= & 2 A_{p_{1}} A_{p_{2}} \exp \left\{j 2 \pi\left[\left(f_{p_{1}}+f_{p_{2}}\right) \tau+\left(\frac{\gamma_{p_{1}}}{2}+\frac{\gamma_{p_{2}}}{2}\right) 2 t \tau\right]\right\} \\
& \cdot \cos \left\{2 \pi\left[\left(f_{p_{1}}-f_{p_{2}}\right) t+\left(\frac{\gamma_{p_{1}}}{2}-\frac{\gamma_{p_{2}}}{2}\right)\left(t^{2}+\tau^{2}\right)\right]\right\}=2 A_{p_{1}} A_{p_{2}} R_{p_{1}, p_{2}, 1}(t, \tau) R_{p_{1}, p_{2}, 2}(t, \tau),
\end{aligned}
$$

where

$$
\begin{gathered}
R_{p_{1}, p_{2}, 1}(t, \tau)=\exp \left\{j 2 \pi\left[\left(f_{p_{1}}+f_{p_{2}}\right) \tau+\left(\frac{\gamma_{p_{1}}}{2}+\frac{\gamma_{p_{2}}}{2}\right) 2 t \tau\right]\right\}, \\
R_{p_{1}, p_{2}, 2}(t, \tau)=\cos \left\{2 \pi\left[\left(f_{p_{1}}-f_{p_{2}}\right) t+\left(\frac{\gamma_{p_{1}}}{2}-\frac{\gamma_{p_{2}}}{2}\right)\left(t^{2}+\tau^{2}\right)\right]\right\} .
\end{gathered}
$$

The cross-term $D_{s, \text { cros }}\left(f_{\tau}, f_{t \tau}, \xi\right)$ can be represented as

$$
D_{s, \operatorname{cros}}\left(f_{\tau}, f_{t \tau}, \xi\right)=2 A_{p_{1}} A_{p_{2}} \operatorname{FFT}_{\tau}\left\{\operatorname{MSFT}_{t \tau}\left[R_{p_{1}, p_{2}, 1}(t, \tau) R_{p_{1}, p_{2}, 2}(t, \tau)\right]\right\} .
$$

$1^{\circ}: \gamma_{p_{1}}=\gamma_{p_{2}}$ and $f_{p_{1}} \neq f_{p_{2}}$. Performing FFT and MSFT operators on $R_{p_{1}, p_{2}, 1}(t, \tau)$ and $R_{p_{1}, p_{2}, 2}(t, \tau)$, we obtain

$$
\begin{aligned}
D_{p_{1}, p_{2}, \operatorname{cros}, 1}\left(f_{\tau}, f_{t \tau}, \xi\right) & =\operatorname{FFT}_{\tau}\left(\operatorname{MSFT}_{t \tau}\left\{\exp \left[j 2 \pi\left(\nabla f_{p_{1}, p_{2}} \tau+\nabla \gamma_{p_{1}, p_{2}} 2 t \tau\right)\right]\right\}\right) \\
=\delta\left(f_{\tau}-\nabla f_{p_{1}, p_{2}}\right) \delta\left(f_{t \tau}-\frac{2 \nabla \gamma_{p_{1}, p_{2}}}{\xi}\right) & \\
D_{p_{1}, p_{2}, \operatorname{cros}, 2}\left(f_{\tau}, f_{t \tau}, \xi\right) & =\operatorname{FFT}_{\tau}\left\{\operatorname{MSFT}_{t \tau}\left[\cos \left(2 \pi \Delta f_{p_{1}, p_{2}} t\right)\right]\right\} \\
& =\frac{\Delta f_{p_{1}, p_{2}}}{\xi f_{t \tau}^{2}} \cos \left(2 \pi \frac{\Delta f_{p_{1}, p_{2}}}{\xi f_{t \tau}} f_{\tau}\right) .
\end{aligned}
$$


Combining Eqs. (23) and (24) and exploiting the two-dimensional (2-D) convolution theorem lead to the following:

1. If $\tilde{f}_{t \tau}=0$, we obtain

$$
D_{s, \operatorname{cros}}\left(f_{\tau}, f_{t \tau}, \xi\right)=0 .
$$

2. If $\tilde{f}_{t \tau} \neq 0$, we obtain

$$
D_{s, \operatorname{cros}}\left(f_{\tau}, f_{t \tau}, \xi\right)=2 A_{p_{1}} A_{p_{2}} \frac{\Delta f_{p_{1}, p_{2}}}{\xi \tilde{f}_{t \tau}^{2}} \cos \left(2 \pi \frac{\Delta f_{p_{1}, p_{2}}}{\xi \tilde{f}_{t \tau}} \tilde{f}_{\tau}\right) .
$$

$2^{\circ}: \gamma_{p_{1}} \neq \gamma_{p_{2}} . D_{p_{1}, p_{2}, \text { cros }, 1}\left(f_{\tau}, f_{t \tau}, \xi\right)$ is the same as Eq. (23).

As with the analyses in the Lv's distribution, ${ }^{25}$ the principle of the stationary phase is utilized.

$$
\begin{gathered}
D_{p_{1}, p_{2}, \operatorname{cros}, 2}\left(f_{t \tau}, f_{\tau}, \xi\right)=D_{s, \text { cros }, 2}^{\prime \prime}\left(f_{t \tau}, f_{\tau}, \xi\right)+D_{s, \text { cros }, 2}^{\prime \prime}\left(f_{t \tau}, f_{\tau}, \xi\right)=\frac{4 \xi\left|f_{\tau} \Delta \gamma_{p_{1}, p_{2}}\right|}{\left|\xi^{2} f_{t \tau}^{2}+\Delta \gamma_{p_{1}, p_{2}}^{2}\right|^{3 / 2}} \\
\cdot \cos \left\{\operatorname{sgn}\left(\Delta \gamma_{p_{1}, p_{2}}\right) \frac{\pi}{4}\left[1+\operatorname{sgn}\left(\xi^{2} f_{t \tau}^{2}+4 \Delta \gamma_{p_{1}, p_{2}}^{2}\right)\right]+\frac{2 f_{\tau}^{2} \Delta \gamma_{p_{1}, p_{2}}}{\xi^{2} f_{t \tau}^{2}+\Delta \gamma_{p_{1}, p_{2}}^{2}}-\frac{\Delta f_{p_{1}, p_{2}}^{2}}{2 \Delta \gamma_{p_{1}, p_{2}}}\right\} .
\end{gathered}
$$

Then the 2-D convolution theorem is utilized to obtain

$$
\begin{aligned}
& D_{s, \text { cros }}\left(f_{t \tau}, f_{\tau}, \xi\right)=\frac{8 A_{p_{1}} A_{p_{2}} \xi\left|\tilde{f}_{\tau} \Delta \gamma_{p_{1}, p_{2}}\right|}{\left|\xi^{2} \tilde{f}_{t \tau}^{2}+\Delta \gamma_{p_{1}, p_{2}}^{2}\right|^{3 / 2}} \\
& \quad \cdot \cos \left\{\operatorname{sgn}\left(\Delta \gamma_{p_{1}, p_{2}}\right) \frac{\pi}{4}\left[1+\operatorname{sgn}\left(\xi^{2} \tilde{f}_{t}^{2}+\Delta \gamma_{p_{1}, p_{2}}^{2}\right)\right]+\frac{2 \tilde{f}_{\tau}^{2} \Delta \gamma_{p_{1}, p_{2}}}{\xi^{2} \tilde{f}_{t \tau}^{2}+\Delta \gamma_{p_{1}, p_{2}}^{2}}-\frac{\Delta f_{p_{1}, p_{2}}^{2}}{2 \Delta \gamma_{p_{1}, p_{2}}}\right\} .
\end{aligned}
$$

Above is the proof of the lemma listed in Sec. 2.2.

\section{Acknowledgments}

We acknowledge support from National Natural Science Foundation of China (No. 61001204), the Science and Technology Foundation of Shaanxi Province (No. 2012JM8015), and the Xi' an Polytechnic University Dr Support Foundation (No. BS1119).

\section{References}

1. M. Prickett and C. Chen, "Principles of inverse synthetic aperture radar ISAR imaging," in Proc. of Electronics and Aerospace Systems Conf., pp. 340-345 (1980).

2. F. Berizzi et al., "High-resolution ISAR imaging of maneuvering targets by means of the range instantaneous Doppler technique: modeling and performance analysis," IEEE Trans. Image Process. 10(12), 1880-1890 (2001).

3. J. Zheng et al., "ISAR imaging of non-uniformly rotating target based on a fast parameter estimation algorithm of cubic phase signal," IEEE Trans. Geosci. Remote Sens. 53(9), 4727-4740 (2015).

4. H. Zhao et al., "Hierarchical vessel classifier based on multifeature joint matching for highresolution inverse synthetic aperture radar images," J. Appl. Remote Sens. 8, 083563 (2014).

5. M. Xing, R. Wu, and Z. Bao, "High resolution ISAR imaging of high speed moving targets," IEE Proc., Radar Sonar Navig. 152(2), 58-67 (2005).

6. J. Wang and D. Kasilingam, "Global range alignment for ISAR," IEEE Trans. Aerosp. Electron. Syst. 39(1), 351-357 (2003).

7. D. Zhu, Y. Li, and Z. Zhu, "A keystone transform without interpolation for SAR ground moving-target imaging," IEEE Geosci. Remote Sens. Lett. 4(1), 18-22 (2007).

8. M. Xing et al., "Migration through resolution cell compensation in ISAR imaging," IEEE Geosci. Remote Sens. Lett. 1(2), 141-144 (2004). 
9. D. E. Wahl, P. H. Eichel, and D. C. Ghigetia, "Phase gradient autofocus-a robust tool for high resolution SAR phase correction," IEEE Trans. Aerosp. Electron. Syst. 30(3), 827-835 (1994).

10. X. Li, G. Liu, and J. Ni, "Autofocusing of ISAR imaging based on entropy minimization," IEEE Trans. Aerosp. Electron. Syst. 35(4), 1240-1251 (1999).

11. M. Xing et al., "New ISAR imaging algorithm based on modified Wigner-Ville distribution," IET Radar Sonar Navig. 3(1), 70-80 (2009).

12. X. Lv et al., "ISAR imaging of maneuvering targets based on the range centroid Doppler technique," IEEE Trans. Image Process. 47(2), 141-153 (2010).

13. Z. Gao et al., "ISAR imaging of maneuvering targets with the range instantaneous chirp rate technique," IET Radar Sonar Navig. 3(5), 449-460 (2009).

14. Y. Wang and Y. Lin, "ISAR imaging of non-uniformly rotating target via range-instantaneous-Doppler-derivatives algorithm," IEEE J. Sel. Topics Appl. Earth Obs. Remote Sens. 7(1), 167-176 (2014).

15. J. Zheng et al., "An algorithm for phase parameters estimation of multi-chirp signals," $J$. Xi'an Jiaotong Univ. 47(2), 69-74 (2013).

16. V. Chen and S. Qian, "Joint time-frequency transform for radar range-Doppler imaging," IEEE Trans. Aerosp. Electron. Syst. 34(2), 486-499 (1998).

17. P. O'Shea, "A new technique for instantaneous frequency rate estimation," IEEE Signal Process. Lett. 9(8), 251-252 (2002).

18. P. O'Shea, "A fast algorithm for estimating the parameters of a quadratic FM signal," IEEE Trans. Signal Process. 52(2), 385-393 (2004).

19. S. Stein, "Algorithms for ambiguity function processing," IEEE Trans. Acoust. Speech Signal Process. 29(3), 588-599 (1981).

20. P. Wang et al., "Integrated cubic phase function for linear FM signal analysis," IEEE Trans. Aerosp. Electron. Syst. 46(3), 963-977 (2010).

21. M. Wang, A. Chen, and C. Chui, "Linear frequency-modulated signal detection using Radon-ambiguity transform," IEEE Trans. Signal Process. 46(3), 571-586 (1998).

22. X. Lv et al., "Keystone transform of the Wigner-Ville distribution for analysis of multicomponent LFM signals," Signal Process. 89(5), 791-806 (2009).

23. R. Tao, N. Zhang, and Y. Wang, "Analyzing and compensating effects of range and Doppler frequency migrations in linear frequency modulation pulse compression radar," IET Radar Sonar Navig. 5(1), 12-22 (2011).

24. H. Sun et al., "Application of the fractional Fourier transform to moving target detection in airborne SAR,” IEEE Trans. Aerosp. Electron. Syst. 38(4), 1416-1424 (2002).

25. X. Guo et al., "Comments on discrete chirp-Fourier transform and its application to chirp rate estimation," IEEE Trans. Signal Process. 50(12), 3115-3115 (2002).

26. X. Xia, "Discrete chirp-Fourier transform and its application to chirp rate estimation," IEEE Trans. Signal Process. 48(11), 3122-3133 (2000).

27. X. Lv et al., "Lv's distribution: principle, implementation, properties, and performance," IEEE Trans. Signal Process. 59(8), 3576-3591 (2011).

28. S. Luo et al., "Performance analysis on Lv distribution and its applications," Digital Signal Process. 23(3), 797-807 (2013).

29. J. Xu et al., "Radon-Fourier transform (RFT) for radar target detection (I): generalized Doppler filter bank," IEEE Trans. Aerosp. Electron. Syst. 47(2), 1186-1202 (2011).

30. J. Xu et al., "Radon-Fourier transform (RFT) for radar target detection (II): blind speed sidelobe suppression," IEEE Trans. Aerosp. Electron. Syst. 47(4), 2473-2489 (2011).

31. J. Xu et al., "Radar maneuvering target motion estimation based on generalized RadonFourier transform,” IEEE Trans. Signal Process. 60(12), 6190-6201 (2012).

32. J. Zheng et al., "Radar high-speed target detection based on the scaled inverse Fourier transform," IEEE J. Sel. Topics Appl. Earth Obs. Remote Sens. 8(3), 1108-1119 (2015).

33. S. Barbarossa, "Analysis of multicomponent LFM signals by a combined Wigner-Hough transform," IEEE Trans. Signal Process. 43(6), 1511-1515 (1995).

34. X. Bai et al., "ISAR imaging of a ship target based on parameter estimation of multicomponent quadratic frequency-modulated signals," IEEE Trans. Geosci. Remote Sens. 52(2), 1418-1429 (2014). 
35. J. Zheng et al., "ISAR imaging of targets with complex motion based on the keystone timechirp rate distribution," IEEE Geosci. Remote Sens. Lett. 11(7), 1275-1279 (2014).

36. J. Zheng et al., "Fast parameter estimation algorithm for cubic phase signal based on quantifying effects of Doppler frequency shift," Prog. Electromagn. Res. 142, 59-74 (2013).

37. J. Zheng et al., "ISAR imaging of targets with complex motion based on the chirp ratequadratic chirp rate distribution," IEEE Trans. Geosci. Remote Sens. 52(11), 7276-7289 (2014).

38. Y. Wang, "Inverse synthetic aperture radar imaging of maneuvering target based on rangeinstantaneous-Doppler and range-instantaneous-chirp-rate algorithms," IET Radar Sonar Navig. 6(9), 921-928 (2012).

39. Y. Wang, J. Kang, and Y. Jiang, "ISAR imaging of maneuvering target based on the local polynomial Wigner distribution and integrated high-order ambiguity function for cubic phase signal model," IEEE J. Sel. Topics Appl. Earth Obs. Remote Sens. 7(7), 29712991 (2014).

40. S. Peleg and B. Porat, "The Cramer-Rao lower bound for signals with constant amplitude and polynomial phase," IEEE Trans. Aerosp. Electron. Syst. 34(4), 1070-1084 (1998).

41. S. Peleg and B. Porat, "Linear FM signal parameter estimation from discrete-time observations," IEEE Trans. Aerosp. Electron. Syst. 27(4), 607-616 (1991).

42. Y. Li et al., "Inverse synthetic aperture radar imaging of ship target with complex motion," IET Radar Sonar Navig. 2(6), 395-403 (2008).

Li Yanyan received her BS and MS degrees from Xidian University in 2008 and 2011, respectively. Currently, she is working toward her $\mathrm{PhD}$ in the School of Electronic Engineering, Xidian University, Xi' an, China. Her research interests include inverse synthetic aperture radar imaging, target detection, and target tracking.

Su Tao received the BS, MS, and PhD degrees from Xidian University, Xi' an, China, in 1990, 1993, and 1999, respectively. Since 1993, he has been a faculty at Xidian University, where he is currently a professor with the National Laboratory of Radar Signal Processing, School of Electronic Engineering. His research interests include high speed real time signal processing on radar, sonar and telecommunications, digital signal processing, parallel processing system design, and FPGA IP design.

Zheng Jibin received the BS degree in electronic information science and technology from Shandong Normal University, Shandong, China, in 2009, and the PhD degree in signal and information processing from Xidian University in 2015. Since 2015, he has been a faculty at National Laboratory of Radar Signal Processing at Xidian University, Shaanxi, China. His research interests include synthetic aperture radar (SAR) and inverse SAR signal processing, cognitive radar, and time-frequency analysis.

He Xuehui received the BS degree in mathematics from Nanchang University, in 2001, and MS and $\mathrm{PhD}$ degrees in signal and information processing from Xidian University in 2004 and 2010, respectively. Since 2004, he has been a faculty at Xidian University, where he is currently an assitant professor at National Lab of Radar Signal Processing of School of Electronic Engineering. His research interests include the digital signal processing, and the FPGA IP design. 